\title{
Error bounds of regularized gap functions for weak vector variational inequality problems
}

\section{Minghua Li*}

\section{"Correspondence:}

minghuali20021848@163.com

College of Science, Northwest A\&F

University, xinong road, Yangling,

Shaanxi, China

\begin{abstract}
In this paper, by the nonlinear scalarization method, a global error bound of a weak vector variational inequality is established via a regularized gap function. The result extends some existing results in the literature.

MSC: 49K40; $90 \mathrm{C} 31$
\end{abstract}

Keywords: error bound; regularized gap function; weak vector variational inequality

\section{Introduction}

Throughout this paper, let $K$ be a closed convex subset of an Euclidean space $R^{n}$ and $F: R^{n} \rightarrow B\left(R^{n}, R^{m}\right)$ be a continuously differentiable mapping. We consider a weak vector variational inequality (WVVI) of finding $x^{*} \in K$ such that

$$
\left\langle F\left(x^{*}\right), x-x^{*}\right\rangle \notin-\operatorname{int} C, \quad \forall x \in K,
$$

where $C \subseteq R^{m}$ is a closed convex and pointed cone with nonempty interior int $C$. (WVVI) was firstly introduced by Giannessi [1]. It has been shown to have many applications in vector optimization problems and traffic equilibrium problems (e.g., $[2,3])$.

Error bounds are to depict the distance from a feasible solution to the solution set, and have played an important role not only in sensitivity analysis but also in convergence analysis of iterative algorithms. Recently, kinds of error bounds have been presented for weak vector variational inequalities in [4-7]. By using a scalarization approach of Konnov [8], $\mathrm{Li}$ and Mastroeni [5] established the error bounds for two kinds of (WVVIs) with setvalued mappings. By a regularized gap function and a D-gap function for a weak vector variational inequality, Charitha and Dutta [4] obtained the error bounds of (WVVI), respectively. Recently, in virtue of the regularized gap functions, Sun and Chai [6] studied some error bounds for generalized (WVVIs). By using the image space analysis, Xu and Li [7] got a gap function for (WVVI). Then, they established an error bound for (WVVI) without the convexity of the constraint set. These papers have a common characteristic: the solution set of (WVVI) is a singleton [6,7]. Even though the solution set of (WVVI) is not a singleton $[4,5]$, the solution set of the corresponding variational inequality (VI) is a singleton, when their results reduce to (VI).

In this paper, by the nonlinear scalarization method, we study a global error bound of (WVVI). This paper is organized as follows. In Section 2, we establish a global error bound 
of (VI) via the generalized gap functions. In Section 3, we discuss a global error bound of (WVVI) by the nonlinear scalarization method.

\section{A global error bound of (VI)}

Let $h: R^{n} \rightarrow R \cup\{+\infty\}$ be a proper lower semicontinuous function, and let $S=$ $\left\{x \in R^{n} \mid h(x) \leq 0\right\} . h$ has a global error bound if there exists $\tau>0$ such that

$$
d(x, S) \leq \tau h(x)_{+}, \quad \forall x \in X,
$$

where $h(x)_{+}:=\max \{h(x), 0\}$ and $d(x, S):=\inf \{\|x-s\| \mid s \in S\}$ if $S$ is nonempty and $d(x, S)=$ $+\infty$ if $S$ is empty. $f: R^{n} \rightarrow R^{n}$ is said to be coercive on $K$ if

$$
\lim _{x \in K,\|x\| \rightarrow+\infty} \frac{\langle f(x), x-y\rangle}{\|x\|}=+\infty, \quad \forall y \in K .
$$

$f: R^{n} \rightarrow R^{n}$ is said to be strongly monotone on $R^{n}$ with the modulus $\lambda>0$ if

$$
\left\langle f(x)-f\left(x^{\prime}\right), x-x^{\prime}\right\rangle \geq \lambda\left\|x-x^{\prime}\right\|^{2}, \quad \forall x, x^{\prime} \in R^{n} .
$$

In this section, we establish a global error bound of (VI) of finding $x \in K$ such that

$$
\langle f(x), y-x\rangle \geq 0, \quad \forall y \in K
$$

where $f: R^{n} \rightarrow R^{n}$ is a continuously differentiable mapping.

To study the error bound of (VI), we need to construct a class of merit functions which were made to reformulate (VI) as an optimization problem; see [9-16]. One of such functions is a generalized regularized gap function [17] defined by

$$
f_{\gamma}(x):=-\inf _{y \in K}\{\langle f(x), y-x\rangle+\gamma \varphi(x, y)\}, \quad \forall x \in R^{n}, \gamma>0,
$$

where $\varphi: R^{n} \times R^{n} \rightarrow R$ is a real-valued function with the following properties:

(P1) $\varphi$ is continuously differentiable on $R^{n} \times R^{n}$.

(P2) $\varphi(x, y) \geq 0, \forall x, y \in R^{n}$ and the equality holds if and only if $x=y$.

(P3) $\varphi(x, \cdot)$ is uniformly strongly convex on $R^{n}$ with the modulus $\beta>0$ in the sense that

$$
\varphi\left(x, y_{1}\right)-\varphi\left(x, y_{2}\right) \geq\left\langle\nabla_{2} \varphi\left(x, y_{2}\right), y_{1}-y_{2}\right\rangle+\beta\left\|y_{1}-y_{2}\right\|^{2}, \quad \forall x, y_{1}, y_{2} \in R^{n}
$$

where $\nabla_{2} \varphi$ denotes the partial derivative of $\varphi$ with respect to the second variable.

(P4) $\nabla_{2} \varphi(\cdot, y)$ is uniformly Lipschitz continuous on $R^{n}$ with the modulus $\alpha$, i.e., for all $x \in R^{n}$

$$
\left\|\nabla_{2} \varphi\left(x, y_{1}\right)-\nabla_{2} \varphi\left(x, y_{2}\right)\right\| \leq \alpha\left\|y_{1}-y_{2}\right\|, \quad \forall y_{1}, y_{2} \in R^{n} .
$$

Now we recall some properties of $\varphi$ in (1).

Proposition 2.1 The following statements hold for each $x, y \in K$ :

(i) $\left\langle\nabla_{2} \varphi(x, y), u\right\rangle \leq \alpha\|x-y\|\|u\|, \forall u \in \operatorname{span}(K-x)$.

(ii) $\beta\|x-y\|^{2} \leq \varphi(x, y) \leq(\alpha-\beta)\|x-y\|^{2}$. 
(iii) $\varphi(x, y)-\left\langle\nabla_{2} \varphi(x, y), y-x\right\rangle \geq-(\alpha-\beta)\|x-y\|^{2}$.

(iv) $\nabla_{2} \varphi(x, y)=0$ if and only if $x=y$.

Proof Parts (i)-(iii) are taken from [14, Lemma 2.1] and part (iv) is from [17, Lemma 2.1].

Remark 2.1 In light of (ii) in Proposition 2.1, it holds true that $\alpha \geq 2 \beta$.

Then we list some basic properties of the generalized regularized gap function $f_{\gamma}$.

Proposition 2.2 The following conclusions are valid for (VI).

(i) For every $x \in R^{n}$, there exists a unique vector $y_{\gamma}^{\varphi}(x) \in K$ at which the infimum in (1) is attained, i.e.,

$$
f_{\gamma}(x)=-\left\langle f(x), y_{\gamma}^{\varphi}(x)-x\right\rangle-\gamma \varphi\left(x, y_{\gamma}^{\varphi}(x)\right)
$$

(ii) $f_{\gamma}$ is a gap function of $(V I)$.

(iii) $x=y_{\gamma}^{\varphi}(x)$ if and only if $x$ is a solution of $(V I)$.

(iv) $f_{\gamma}$ is continuously differentiable on $R^{n}$ with

$$
\nabla f_{\gamma}(x)=-\nabla f(x)\left(y_{\gamma}^{\varphi}(x)-x\right)+f(x)-\gamma \nabla_{1} \varphi\left(x, y_{\gamma}^{\varphi}(x)\right)
$$

(v) $y_{\gamma}^{\varphi}$ and $f_{\gamma}$ are both locally Lipschitz on $R^{n}$.

(vi) Iff is coercive on $K$, then (VI) has a nonempty compact solution set.

(vii) $f_{\gamma}(x) \geq \beta \gamma\left\|y_{\gamma}^{\varphi}(x)-x\right\|^{2}, \forall x \in K$.

Proof Parts (i)-(iv) are from [16], part (v) from [18, Lemma 3.1] and part (vi) from [11, Proposition 2.2.7].

It follows from (ii) and (iii) that we only need to prove (vii) for $x \in K \backslash S$. Since $y_{\gamma}^{\varphi}(x)$ is the minimizer of the function

$$
G(\cdot):=\langle f(x), \cdot-x\rangle+\gamma \varphi(x, \cdot) \quad \text { on } K
$$

the first-order optimality condition implies that

$$
\left\langle\nabla G\left(y_{\gamma}^{\varphi}(x)\right), y-y_{\gamma}^{\varphi}(x)\right\rangle \geq 0, \quad \forall y \in K
$$

Letting $y=x$, we get

$$
\left\langle\nabla G\left(y_{\gamma}^{\varphi}(x)\right),-y_{\gamma}^{\varphi}(x)+x\right\rangle \geq 0
$$

i.e.,

$$
\left\langle f(x)+\gamma \nabla_{2} \varphi\left(x, y_{\gamma}^{\varphi}(x)\right), y_{\gamma}^{\varphi}(x)-x\right\rangle \leq 0 \text {. }
$$

It follows from (P3) that the mapping $G$ is strongly convex on $R^{n}$ with the modulus $\beta>0$, i.e., $\forall x, y_{1}, y_{2} \in R^{n}$

$$
G\left(x, y_{1}\right)-G\left(x, y_{2}\right) \geq\left\langle f(x)+\gamma \nabla_{2} \varphi\left(x, y_{2}\right), y_{1}-y_{2}\right\rangle+\beta \gamma\left\|y_{1}-y_{2}\right\|^{2} .
$$


Letting $y_{1}=x$ and $y_{2}=y_{\gamma}^{\varphi}(x)$, by $f_{\gamma}(x)=-G\left(x, y_{\gamma}^{\varphi}(x)\right)$, we obtain

$$
f_{\gamma}(x) \geq\left\langle f(x)+\gamma \nabla_{2} \varphi\left(x, y_{\gamma}^{\varphi}(x)\right), x-y_{\gamma}^{\varphi}(x)\right\rangle+\beta \gamma\left\|y_{\gamma}^{\varphi}(x)-x\right\|^{2} .
$$

Thus, one has $f_{\gamma}(x) \geq \beta \gamma\left\|y_{\gamma}^{\varphi}(x)-x\right\|^{2}$.

Theorem 2.1 Let $f$ be coercive on $K$ and $\gamma(\alpha-2 \beta)<\mu$. Assume that $\varphi$ satisfies

(P5) $\left\langle\nabla_{1} \varphi\left(x, y_{\gamma}^{\varphi}(x)\right)+\nabla_{2} \varphi\left(x, y_{\gamma}^{\varphi}(x)\right), y_{\gamma}^{\varphi}(x)-x\right\rangle \geq 0, \forall x \in K$.

Suppose further that the following condition holds:

$$
\mu:=\inf \left\{\langle d, \nabla f(x) d\rangle \mid x \in K \backslash S, d=\frac{y_{\gamma}^{\varphi}(x)-x}{\left\|y_{\gamma}^{\varphi}(x)-x\right\|}\right\}>0,
$$

where $S$ is the solution set of $(V I)$. Then $\sqrt{f_{\gamma}}$ has a global error bound with the modulus

$$
\max \left\{\frac{2 \sqrt{\beta \gamma}}{\mu+2 \gamma \beta-\gamma \alpha}, \frac{2 \sqrt{\beta \gamma}}{\beta \gamma}\right\} .
$$

Proof It follows from (vi) of Proposition 2.2 that $S$ is a nonempty compact set of $K$. If $x \in S$, then the assertion obviously holds. Let $x \in K \backslash S$. Then $f_{\gamma}(x)>0$. For brevity, we denote $w:=y_{\gamma}^{\varphi}(x)-x$ and $d:=\frac{w}{\|w\|}$. It follows from [19, Theorem 2.5] that we only need to prove

$$
\nabla \sqrt{f_{\gamma}}(x) d \leq-\min \left\{\frac{\mu+2 \gamma \beta-\gamma \alpha}{2 \sqrt{\beta \gamma}}, \frac{\sqrt{\beta \gamma}}{2}\right\} .
$$

It follows from (iv) of Proposition 2.2 that

$$
\begin{aligned}
\nabla f_{\gamma}(x) w= & \left\langle-\nabla f(x) w+f(x)-\gamma \nabla_{1} \varphi\left(x, y_{\gamma}^{\varphi}(x)\right), w\right\rangle \\
= & \langle-\nabla f(x) w, w\rangle+\langle f(x), w\rangle+\gamma \varphi\left(x, y_{\gamma}^{\varphi}(x)\right) \\
& -\gamma\left[\left\langle\nabla_{1} \varphi\left(x, y_{\gamma}^{\varphi}(x)\right), w\right\rangle+\varphi\left(x, y_{\gamma}^{\varphi}(x)\right)\right] \\
= & \langle-\nabla f(x) w, w\rangle-f_{\gamma}(x)-\gamma\left[\left\langle\nabla_{1} \varphi\left(x, y_{\gamma}^{\varphi}(x)\right), w\right\rangle+\varphi\left(x, y_{\gamma}^{\varphi}(x)\right)\right] .
\end{aligned}
$$

By (P5) and (2), we have

$$
\nabla f_{\gamma}(x) w \leq-\mu\|w\|^{2}-f_{\gamma}(x)-\gamma\left[-\left\langle\nabla_{2} \varphi\left(x, y_{\gamma}^{\varphi}(x)\right), w\right\rangle+\varphi\left(x, y_{\gamma}^{\varphi}(x)\right)\right] .
$$

It follows from (iii) of Proposition 2.1 that

$$
\nabla f_{\gamma}(x) w \leq-\mu\|w\|^{2}-f_{\gamma}(x)+\gamma(\alpha-\beta)\|w\|^{2} .
$$

Thus,

$$
\nabla \sqrt{f_{\gamma}}(x) d=\frac{\nabla f_{\gamma}(x) \frac{w}{\|w\|}}{2 \sqrt{f_{\gamma}(x)}} \leq \frac{-[\mu-\gamma(\alpha-\beta)]\|w\|}{2 \sqrt{f_{\gamma}(x)}}-\frac{\sqrt{f_{\gamma}(x)}}{2\|w\|} .
$$

In light of (4) and (vii) of Proposition 2.2, we have

$$
\nabla \sqrt{f_{\gamma}}(x) d \leq-\frac{[\mu-\gamma(\alpha-\beta)]\|w\|}{2 \sqrt{f_{\gamma}(x)}}-\frac{\sqrt{\beta \gamma}}{2} .
$$


If $\mu<\gamma(\alpha-\beta)$, then it follows from $\gamma(\alpha-2 \beta)<\mu$ that

$$
\nabla \sqrt{f_{\gamma}}(x) d \leq \frac{[\gamma(\alpha-\beta)-\mu]}{2 \sqrt{\beta \gamma}}-\frac{\sqrt{\beta \gamma}}{2}=\frac{\gamma \alpha-\mu-2 \gamma \beta}{2 \sqrt{\beta \gamma}}<0 .
$$

If $\mu \geq \gamma(\alpha-\beta)$, then

$$
\nabla \sqrt{f_{\gamma}}(x) d \leq-\frac{\sqrt{\beta \gamma}}{2}
$$

Hence, (3) follows from (5) and (6). The proof is complete.

Now we use two examples to show that (2) cannot be dropped and that Theorem 2.1 is applicable, respectively.

Example 2.1 Consider $K=R, \varphi(x, y)=\frac{1}{2}|x-y|^{2}, \gamma=\frac{1}{2}$ and $f(x)=x^{3}$. Then we can easily get that $\alpha=2 \beta=1, y_{\gamma}^{\varphi}(x)=x-2 f(x), f_{\gamma}(x)=x^{6}$ and $S=\{0\}$. It is clear that $f$ is coercive on $K$ and $\mu=0$. Thus, (2) does not hold. Moreover, it is obvious that $\sqrt{f_{\gamma}}$ does not have a global error bound.

Example 2.2 Consider $K=[0,+\infty), \varphi(x, y)=\frac{1}{2}|x-y|^{2}, \gamma=1$ and $f(x)=x$. Then we can easily get that $\alpha=2 \beta=1, \nabla f(x)=1, y_{\gamma}^{\varphi}(x)=0, f_{\gamma}(x)=\frac{1}{2} x^{2}$ and $S=\{0\}$. It is clear that $f$ is coercive on $K$ and (2) holds. Thus, it follows from Theorem 2.1 that $\sqrt{f_{\gamma}}$ has a global error bound.

By [21, Proposition 2.3(ii)] Huang and $\mathrm{Ng}$ [14, Theorem 2.1] have obtained the following conclusion. Now we give a slightly different proof by Theorem 2.1.

Corollary 2.1 Let $f$ be strongly monotone on $R^{n}$ with the modulus $\lambda>0$ and $\gamma(\alpha-2 \beta)<\lambda$. Assume that $\varphi$ satisfies (P5). Then $\sqrt{f_{\gamma}}$ has a global error bound with the modulus

$$
\max \left\{\frac{2 \sqrt{\beta \gamma}}{\lambda+2 \gamma \beta-\gamma \alpha}, \frac{2 \sqrt{\beta \gamma}}{\beta \gamma}\right\}
$$

Proof Let $x \in K \backslash S$ and $w=y_{\gamma}^{\varphi}(x)-x$. Since $f$ is continuously differentiable, then

$$
f(x+t w)=f(x)+t\langle\nabla f(x), w\rangle+o(t)
$$

where $\frac{o(t)}{t} \rightarrow 0$ as $t \rightarrow 0$. Since $f$ is strongly monotone with the modulus $\lambda$, one has

$$
\langle f(x+t w)-f(x), t w\rangle \geq \lambda\|t w\|^{2},
$$

which implies that

$$
\langle w, \nabla f(x) w\rangle \geq \lambda\|w\|^{2}
$$

Thus, (2) holds. Moreover, the strong monotonicity of $f$ implies the coerciveness of $f$ ( $c f$. [22, Remark 2.1]). Thus, by Theorem 2.1, we get that $\sqrt{f_{\gamma}}$ has a global error bound. 


\section{A global error bound of (WVVI)}

In this section, by the nonlinear scalarization method and by Theorem 2.1, we discuss a global error bound of (WVVI). The dual cone of $C$ is defined by $C^{*}:=\left\{\xi \in R^{m}\right.$ : $\langle\xi, z\rangle \geq 0, \forall z \in C\}$. For each $\xi \in R^{m},\|\xi\|:=\sup \{|\langle\xi, z\rangle|:\|z\| \leq 1\}$, where $\langle\xi, z\rangle$ denotes the value of $\xi$ at $z$. Let $e \in \operatorname{int} C$ and $B_{e}^{*}:=\left\{\xi \in C^{*}:\langle\xi, e\rangle=1\right\}$. It is well known that $B_{e}^{*}$ is a compact convex base of $C^{*}$.

Lemma $3.1[20]$

$$
S \supset \bigcup_{\xi \in C^{*} \backslash\{0\}} S_{\xi}=\bigcup_{\xi \in B_{e}^{*}} S_{\xi},
$$

where $S_{\xi}:=\left\{x \in K:\left\langle\sum_{i=1}^{m} \xi_{i} F_{i}\left(x^{*}\right), x-x^{*}\right\rangle \geq 0, \forall y \in K\right\}$ and $S$ is the solution set of (WVVI).

Recall the generalized regularized gap function for (WVVI) which is defined by

$$
\phi_{\gamma}(x):=\min _{\xi \in B_{e}^{*}} f_{\gamma}(x, \xi)
$$

where $f_{\gamma}(x, \xi)=\max _{y \in K}\left\{\left\langle\sum_{i=1}^{m} \xi_{i} F_{i}(x), x-y\right\rangle-\gamma \varphi(x, y)\right\}$. When $\phi(x, y)=\frac{1}{2}\|x-y\|^{2}$, the generalized regularized gap function reduces to the regularized gap function which was defined in [4].

Theorem 3.1 Let $\gamma(\alpha-2 \beta)<\min _{\xi \in B_{e}^{*}} \mu_{\xi}$. Assume that $\varphi$ satisfies (P5). For each $\xi \in B_{e}^{*}$, suppose that $\xi \circ F$ is coercive on $K$, and that the following condition holds:

$$
\mu_{\xi}:=\inf \left\{\langle d,(\xi \circ \nabla F)(x) d\rangle \mid x \in K \backslash S, d=\frac{y_{\gamma}^{\varphi}(x)-x}{\left\|y_{\gamma}^{\varphi}(x)-x\right\|}\right\}>0
$$

Then $\sqrt{\phi_{\gamma}}$ has a global error bound with the modulus

$$
\max \left\{\max _{\xi \in B_{e}^{*}} \frac{2 \sqrt{\beta \gamma}}{\mu_{\xi}+2 \gamma \beta-\gamma \alpha}, \frac{2 \sqrt{\beta \gamma}}{\beta \gamma}\right\} .
$$

Proof It follows from (vi) of Proposition 2.2 that $S_{\xi}$ is a nonempty compact set of $K$ for each $\xi \in B_{e}^{*}$. If $x \in S$, then the assertion obviously holds. Let $x \in K \backslash S$. Then $\phi_{\gamma}(x)>0$ and there exists $\xi_{0} \in B_{e}^{*}$ such that $f_{\gamma}\left(x, \xi_{0}\right)=\phi_{\gamma}(x)$. It follows from Theorem 2.1 that

$$
d\left(x, S_{\xi_{0}}\right) \leq \tau_{\xi_{0}} \sqrt{f_{\gamma}\left(x, \xi_{0}\right)},
$$

where $\tau_{\xi}=\max \left\{\frac{2 \sqrt{\beta \gamma}}{\mu_{\xi}+2 \gamma \beta-\gamma \alpha}, \frac{2 \sqrt{\beta \gamma}}{\beta \gamma}\right\}$. Thus, by Lemma 3.1, one has

$$
d(x, S) \leq d\left(x, S_{\xi_{0}}\right) \leq \tau_{\xi_{0}} \cdot \sqrt{f_{\gamma}\left(x, \xi_{0}\right)} \leq \max _{\xi \in B_{e}^{*}} \tau_{\xi} \cdot \sqrt{\phi_{\gamma}(x)} .
$$

Hence, $\sqrt{\phi_{\gamma}}$ has a global error bound with the modulus $\max _{\xi \in B_{e}^{*}} \tau_{\xi}$. 
Remark 3.1 If $F_{i}$ is strongly monotone with the modulus $\lambda_{i}$ for $i=1,2, \ldots, m$ and $C=R_{+}^{m}$, it follows from [21, Proposition 2.3] that

$$
\langle d,(\xi \circ \nabla F)(x) d\rangle \geq \lambda\|d\|^{2}, \quad \forall d \in R^{n}, \xi \in B_{e}^{*} .
$$

Moreover, the strong monotonicity of $F_{i}$ implies the coerciveness of $F_{i}(c f$. [22, Remark 2.1]) and that (VI) has a unique solution (cf. [11, Theorem 2.3.3]). Thus, by Theorem 3.1, we get that $\sqrt{\phi_{\gamma}}$ has a global error bound. Hence, our results extend those of [4, Theorem 2.9].

\section{Competing interests}

The author declares that he has no competing interests.

\section{Acknowledgements}

This research was supported by the Natural Science Foundation of Shaanxi Province, China (Grant number: 2014JQ1023).

Received: 20 June 2014 Accepted: 18 August 2014 Published: 2 September 2014

\section{References}

1. Giannessi, F: Theorems of the alternative, quadratic programs and complementarity problems. In: Cottle, RW, Giannessi, F, Lions, JL (eds.) Variational Inequalities and Complementarity Problems, pp. 151-186. Wiley, New York (1980)

2. Chen, GY, Goh, CJ, Yang, XQ: Vector network equilibrium problems and nonlinear scalarization methods. Math. Methods Oper. Res. 49, 239-253 (1999)

3. Li, SJ, Teo, KL, Yang, XQ: A remark on a standard and linear vector network equilibrium problem with capacity constraints. Eur. J. Oper. Res. 184, 13-23 (2008)

4. Charitha, C, Dutta, J: Regularized gap functions and error bounds for vector variational inequalities. Pac. J. Optim. 6, 497-510(2010)

5. Li, J, Mastroeni, G: Vector variational inequalities involving set-valued mappings via scalarization with applications to error bounds for gap functions. J. Optim. Theory Appl. 145, 355-372 (2010)

6. Sun, XK, Chai, Y: Gap functions and error bounds for generalized vector variational inequalities. Optim. Lett. 8 , 1663-1673 (2014)

7. $\mathrm{Xu}, \mathrm{YD}, \mathrm{Li}, \mathrm{SJ}$ : Gap functions and error bounds for weak vector variational inequalities. Optimization 63, 1339-1352 (2014)

8. Konnov, IV: A scalarization approach for vector variational inequalities with applications. J. Glob. Optim. 32, 517-527 (2005)

9. Auchmuty, G: Variational principles for variational inequalities. Numer. Funct. Anal. Optim. 10, 863-874 (1989)

10. Auslender, A: Optimization, Méthodes Numériques. Masson, Paris (1976)

11. Facchinei, F, Pang, JS: Finite-Dimensional Variational Inequalities and Complementarity Problems. Springer, Berlin (2003)

12. Fukushima, M: Equivalent differentiable optimization problems and descent methods for asymmetric variational inequality problems. Math. Program. 53, 99-110 (1992)

13. Harker, PT, Pang, JS: Finite-dimensional variational inequality and nonlinear complementarity problems: a survey of theory, algorithms, and applications. Math. Program. 48, 161-220 (1990)

14. Huang, LR, Ng, KF: Equivalent optimization formulations and error bounds for variational inequality problems. J. Optim. Theory Appl. 125, 299-314 (2005)

15. Ng, KF, Tan, LL: Error bounds of regularized gap functions for nonsmooth variational inequality problems. Math. Program. 110, 405-429 (2007)

16. $\mathrm{Wu}, \mathrm{JH}$, Florian, $\mathrm{M}$, Marcotte, $\mathrm{P}$ : A general descent framework for the monotone variational inequality problem. Math. Program. 61, 281-300 (1993)

17. Yamashita, N, Taji, K, Fukushima, M: Unconstrained optimization reformulations of variational inequality problems. J. Optim. Theory Appl. 92, 439-456 (1997)

18. Li, G, Tang, C, Wei, Z: Error bound results for generalized D-gap functions of nonsmooth variational inequality problems. J. Comput. Appl. Math. 233, 2795-2806 (2010)

19. Ng, KF, Zheng, XY: Error bounds for lower semicontinuous functions in normed spaces. SIAM J. Optim. 12, 1-17 (2001)

20. Lee, GM, Kim, DS, Lee, BS, Yen, ND: Vector variational inequality as a tool for studying vector optimization problems. Nonlinear Anal. 34, 745-765 (1998)

21. Jiang, HY, Qi, LQ: Local uniqueness and convergence of iterative methods for nonsmooth variational inequalities. J. Math. Anal. Appl. 196, 314-331 (1995)

22. Li, G, Ng, KF: Error bounds of generalized D-gap functions for nonsmooth and nonmonotone variational inequality problems. SIAM J. Optim. 20, 667-690 (2009) 